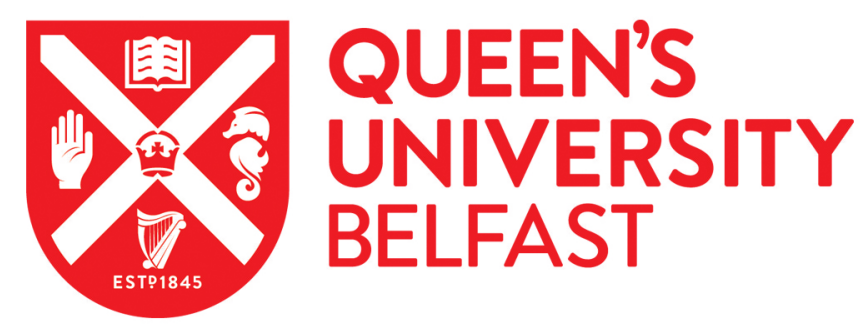

\title{
Protocol : Exploring the Objective Structured Clinical Examination (OSCE) using Institutional Ethnography
}

Kearney, G., Johnston, J., Hart, N., Corman , M., \& Gormley, G. (2018). Protocol : Exploring the Objective Structured Clinical Examination (OSCE) using Institutional Ethnography. International Journal of Educational Research. https://doi.org/10.1016/j.ijer.2018.01.002

Published in:

International Journal of Educational Research

Document Version:

Peer reviewed version

Queen's University Belfast - Research Portal:

Link to publication record in Queen's University Belfast Research Portal

\section{Publisher rights}

Copyright 2018 Elsevier.

This manuscript is distributed under a Creative Commons Attribution-NonCommercial-NoDerivs License

(https://creativecommons.org/licenses/by-nc-nd/4.0/), which permits distribution and reproduction for non-commercial purposes, provided the author and source are cited

\section{General rights}

Copyright for the publications made accessible via the Queen's University Belfast Research Portal is retained by the author(s) and / or other copyright owners and it is a condition of accessing these publications that users recognise and abide by the legal requirements associated with these rights.

Take down policy

The Research Portal is Queen's institutional repository that provides access to Queen's research output. Every effort has been made to ensure that content in the Research Portal does not infringe any person's rights, or applicable UK laws. If you discover content in the Research Portal that you believe breaches copyright or violates any law, please contact openaccess@qub.ac.uk. 


\title{
Protocol: Exploring the Objective Structured Clinical Examination (OSCE) using Institutional Ethnography
}

\begin{abstract}
Throughout education, whether of children or student health professionals, the universal emphasis on accountability has opened assessment to increased scrutiny and tension. This Protocol explores the Objective Structured Clinical Examination or OSCE, the dominant assessment tool used throughout health profession education (HPE), to deem students "competent" in clinical and professional skills. Its use has flourished despite concerns in the literature about its unintentional and undesirable effects on future healthcare professionals.

Education and HPE research have been slow to introduce Institutional Ethnography (IE) to the qualitative researcher's toolkit. IE can offer deep insights and potential for change in complex social challenges threatening education and HPE; this introduction and broad explanation of IE offers an alternative framework to plan further innovative studies.
\end{abstract}

\section{Background}

The ubiquitous statement "assessment drives learning" is credited to George Miller (Zaidi and Nasir, 2015 p.109) but its reach goes far beyond that of medical schools; it is a theme that is associated with all aspects of Education. Another common discourse, regardless of the context of the learning, is that of globalisation and the resulting culture of assessment, with strong emphasis on accountability and governance. In the emerging research field that is Health Profession Education, scholars draw on more established disciplines like Education in order to try to address some common tensions, such as how best to assess individuals as "competent" (Shepard, 2000). Educational research has recently taken a critical look inwards, grappling with issues such as standardisation and objectivity in testing. There has been a move away from the psychometric era to take "a social turn" and understand assessment as a social practice (Shay, 2008). Health Professional Education, in more recent times is also placing increasing emphasis on the social aspects, or lack thereof involved in training future clinicians.

Evidence emerging within the international Health Profession Education (HPE) literature expresses concerns about a dominant assessment method used throughout HPE, the Objective Structured Clinical Examination or OSCE. In OSCEs, students rotate through a number of time-limited stations, in which each station is testing a particular clinical skill or professional competence in a simulated environment. Introduced nearly forty year ago, the OSCE was rapidly adopted in medical schools worldwide, later branching into other areas of HPE such as physiotherapy, pharmacy and nursing; it now forms part of many high-stakes post-graduate examinations, including for membership of the Royal College of Physicians in the United Kingdom (Khan et al, 2013). There is also evidence of the use of the OSCEs outside health care, such as the training of police. Furthermore, and exemplifying the need for an in-depth understanding of this important area of HPE, the General Medical Council 
(GMC) plans to implement for the first time, a UK-wide Medical Licencing Assessment (MLA) in 2022 - an important component of final examinations in all UK medical schools - of which the use of

OSCEs to test Clinical and Professional Skills is being consulted on.

Since the introduction and almost universal deployment of the OSCE exam format, a vast amount of research has followed, largely concentrating on further improvements in standardisation and objectivity with a specific focus on fine-tuning the psychometrics. The intention of much of this work has been to produce defensible examinations in keeping with the governance practices in assessment expected of universities and other professional bodies. However, running alongside this dominant trajectory is a line of critical thinking about OSCEs which expresses concerns about the influence of the OSCE on the direction of student education and student behaviour. There is concern that this exam structure is associated with unintended and even undesirable consequences such as "tick-box" style of questioning by students or artificial separation of the knowledge and skills required by health professionals. Critics suggest that "assessment drives learning, but we argue that OSCES ... may be driving learning in the wrong direction" (Gormley et al, 2016) suggesting that the push for psychometric perfection is perhaps ignoring the requirements of HPE to instil the "social aspects" and human relationships required in training health professionals; that OSCEs fail to address the humanistic dimensions of health care.

This study will attempt to gain a deeper understanding of the social practice of the OSCEs and to question whose interests are being served in this quest for objective and defensible examinations which permit students the required licence to be clinicians. It is hoped that this study will offer some powerful insights through the collection of qualitative data in this ongoing and important debate, which ultimately will better inform the HPE community about the influences that this repeated method of assessment may have on the behaviour of the clinicians that are borne out of it and fundamentally question how useful it is for measuring and/or facilitating patient-centred care.

\section{Approach to inquiry - Institutional Ethnography}

This proposed study will be an Institutional Ethnography (IE); a sociological approach to qualitative inquiry that is characterised by its emergent study design where the emphasis is on exploration and discovery. Institutional Ethnography was developed by Dorothy Smith, an English born Canadian scholar; her thinking was informed by the feminist movement and critical theorists, such as Marx, Foucault, Mead, Garfinkel, Bakhtin and Volosinov (see Smith, 2005).

"Institutional ethnography begins where people are and proceeds to discover the workings of the social structure that extends beyond any one of us." (Smith, 2006, p. 3).

The starting point for this form of inquiry is on the ground, observing and talking to people about their work process - what they do - broadly conceived (Smith, 1987). It then importantly moves on to how their work is organised and coordinated within the institution; this secondary focus allows information to be gathered which may not always be known by those working on the ground (Smith, 2005). A defining feature of IE is the mediating role that texts play in this coordination of work 
processes; texts are viewed as being at the juncture between locally lived experience and the institutional relations that organise those experiences (Campbell and Gregor, 2002).

Institutional Ethnography has been used to research issues of social justice in health care (see for example Corman, 2017; Diamond, 1992; Rankin and Campbell, 2006) but is relatively new in education and in health profession education research. This approach to inquiry has been chosen for this project as its intention is to go beyond being critical to being transformative and produce impactful improvements. IE has often been used in research that involves complex social phenomena, to help bring out the tensions, assumptions and unspoken priorities faced by those on the ground; empowering them in a way that helps enable meaningful social change. Exploring the behind the scenes work of the OSCEs will hopefully allow those involved to consider different approaches to their work and challenge the unquestioning reliance on the OSCE.

\section{Research Plan}

\subsection{Research setting}

The setting for this qualitative study will be the summative "Finals" OSCE assessment, which if passed, in combination with Multiple Choice Questions on a separate date, allow graduation from the undergraduate medical degree programme in XXX and a provisional licence by the General Medical Council to work within the National Health Service as a Foundation (Junior) Doctor. These Final MB OSCEs take place over three days, with each student undertaking one set each day lasting just over an hour, with different material on each day. The data collection will take place within the Centre for Medical Education (CME) including the Clinical Skills Education Centre, where students are both taught and examined in clinical skills, meeting rooms and other teaching venues within the centre. Exclusion criteria, where data will not be collected, are at resit exams, at meetings for failing students, at special circumstances meetings (for example in the case of sickness) or at serious concern meetings which are called when a student displays unprofessional behaviour. These exclusion criteria were put in place because the students concerned are present at these meetings and the intention is not to add to the stress of the student on these occasions.

\subsection{Research objectives}

To explore and explicate the social organisation of undergraduate final year OSCEs in medicine.

To create a (social) map of what individuals do on the ground to show how this work central to OSCE examinations is organised and coordinated; highlighting any disjunctures between policy and practice.

\subsubsection{Research questions}

What work goes into implementing OSCEs? How do these individuals give meaning to their work? How do they know what to do and why do they do it?

What are the higher-level (institutional) influences on this work? 


\subsection{Participants}

The main group to be studied, the key informants, are those who make up the Final MB Year Assessment Delivery Group (YADG) within CME in XXX; this study begins from their standpoint. This team are the combination of academic clinical staff and administration staff from the Progress and Assessment Office who are responsible for the development throughout the academic year, leading up to and beyond the delivery of the final year OSCEs. Although a wide range of both academic and clinical staff are invited to these meetings, the core team number around ten participants - all these will be approached for consent for data collection in the form of observation and interviews (see below.) In addition, there will be observation of individuals and groups who have some involvement in OSCEs but do not sit as part of these teams both during the preparation for OSCEs and on the day of the examinations. This will include other academic and administration staff, final year medical students, OSCE examiners, standardised patients (SPS) who are involved in teaching and assessment and examination invigilators. This will be discussed further later.

\subsection{Data collection methods}

In IE, it is important to note that the process is necessarily inductive and cannot be planned out fully at the start, as the researcher cannot be sure where the data will take them. This is not a quantitative study and it is not the intention to produce generalisable results but rather to allow the research team to understand the overt and hidden work processes and how these are organised by outside forces.

Data collection will take place over an academic year, from September 17 to June 18, which would be consistent with the usual level of data collection in ethnographic practice, (see Timeline in Section 6). The emergent design of data collection will take three different forms.

\subsubsection{Direct observation as data collection}

Firstly, there will be direct and general observations of people at work in a variety of OSCE processes (see section 3.5.1 and 3.5.2 for fuller explanation of these observations). These observations will be recorded as copious contemporaneous field notes in a notebook; reflecting all aspects of process and will themselves form part of the analytical process. These will be formally written up as full field notes into formal typed documents soon after the observations for that day have taken place (Emmerson, Fretz and Shaw, 2011). These observations will vary widely in time, from a few minutes of conversation up to a two-hour meeting.

\subsubsection{Interviews as data collection}

The second form of data will be interview data, the direction of which will be driven by the observational data; not only will interviews seek to further explore the work processes observed in section 3.4.1 above, both observations and interviews will lead the researcher to other individuals whose work may need to be explored (see section 3.5 and 3.5.3.) The goal of these interviews will be to build on the observations by speaking to individuals about their different work processes in 
relation to the organisation of the OSCEs. Whilst a rough topic guide for these interviews has been drawn up, interviews in IE often flow along a more conversational style about what the person does and how they know to do it. It is anticipated that these interviews may last up to an hour. It may be necessary to interview individuals more than once as the study proceeds.

\subsubsection{Texts as data collection}

The final form of data will be texts; important texts will be identified and analysed based on the observations and interviews. These texts can be any written information that is used to inform the OSCE process, and with the emphasis of this study on final year OSCEs and the proposed MLA introduction in UK medical schools, may include such written information as that made available by the GMC. It is important to note that consideration of the role of texts is integral in IE because texts are viewed as major constituents of people's everyday lives, having the ability to organise and coordinate peoples work processes (Campbell and Gregor, 2002).

\subsection{Phases of study}

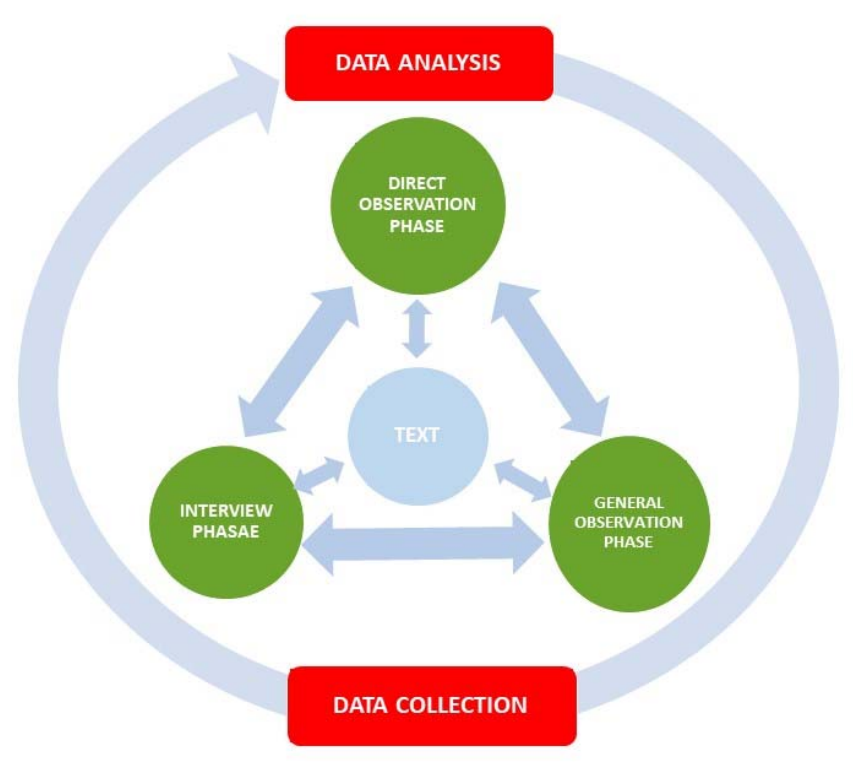

Insert Figure 1. Flow diagram showing the phases of data collection running concurrently with data analysis.

There will be three phases of the study however these phases will run concurrently, in keeping with the emergent design of Institutional Ethnography and be informed by each other. In other words, and outlined in more detail below, while this study begins "on the ground" listening to and observing members of the Final MB YADG as they go about their everyday work, this initial set of observations and interviews will structure who is spoken to next. As Grahame (1999) explains, "The field continuously opens up as the researcher explores the institutional nexus that shapes the local" (cited 
in DeVault and McCoy, 2006, p. 18). As such, what is observed during the initial set of observations and interviews will be central to what is explored during the latter phase of data collection.

\subsubsection{Direct observational/shadowing phase}

In this major part of the study, the research team will shadow the members of the Final MB YADG as they undertake their daily work on the ground preparing for, running and carrying out the processes shaping the final year OSCE examinations throughout the academic year. This shadowing will allow the researcher to fully understand how these people know how to carry out their work which would not be apparent if participants were only interviewed. It will be stressed to these individuals that the purpose of the research is not to judge the quality or integrity of their work, but rather to try to ascertain exactly what they do, how they know to do it, how this is arranged within the institution and what role "texts" have in mediating this. In fact, the position in IE is to advocate for those individuals on the ground and try to look for any tensions they face when carrying out their work. The researcher may informally ask the participants brief questions about what they are doing or how they know what to do at any time. The shadowing will involve the observer being present at all relevant meetings throughout the year where any or all of the Final MB YADG meet with regards to Final MB OSCEs. This will include meetings planning for OSCEs, blueprinting and writing the stations, piloting the stations and following the OSCEs, at the exam board meetings etc. In the case where an individual expresses that they do not want to be involved in direct observations, their wishes will be respected and the process of how to deal with this is laid out in Section 7.

\subsubsection{General Observational Phase}

This phase involves general observations taking place in preparation for and on the day(s) of the OSCE. In advance of the examination days, there will be observation of groups of examiners and SPs as they are trained to take part in the OSCEs. As stated previously, these Final MB OSCEs take place over three days, in six half-day sessions. Individual examiners, SPs and invigilators may be involved in anything from one to all six of these sessions. Examples of what might be observed on the day include the examiner or SP briefings, informal conversations at coffee time between invigilators or the students being corralled after they have finished the examination which is usual practice. These individuals (examiners, SPs, admin staff, invigilators and students) will be emailed in advance of the OSCE day to stress that the observations are about the process of the OSCE and not about any individual's performance on the day. On the case where an individual expresses that they do not want to be involved in these general observations, their wishes will be respected and the process of how to deal with this is also laid out in Section 7. Over the three days of the examinations, the intention will be to observe the breadth of what is going on.

\subsubsection{Interview Phase}

The observational data will open up the field for the semi-structured interviews which will be undertaken alongside and complemented by the observations, as is typical in Institutional Ethnographic studies. These are likely to involve, at a minimum, members of the Final MB YADG but depending on the direction of the data may also include interviewing of other academic and administrative staff, members of the OSCE triad (i.e. examiner, SP and student) and staff involved in 
the immediate process of the OSCE. These will take place on the XXX site at a location and time suitable to the participant.

As is typical for IE, the focus of the interview is not so much the lived experiences of participants, but more about how they know what work they are required to carry out, both formal and informal work and how this work is influenced and organised within the institution. Some of this knowledge may be tacit to the participant and may only emerge through the data collection process. The interviews will be audio recorded using two separate devises and transcribed verbatim, checked for accuracy and fully anonymised for analysis.

\section{Plan for analysis}

Analysis of the material gathered will begin soon after the first observation, supporting the iterative nature of the research. Institutional Ethnography, as an approach to inquiry, is distinctive beyond the conceptual phase of a study and beyond the methods required in data collection. The "ontological shift" put forward in Smith's articulation of Institutional Ethnography orients the ethnographer not only during the field research phase but also during the analysis of the data. Distinctively therefore, the analysis in IE is in two stages; what is happening on the ground, followed by how what is happening is organised outside the control (and sometimes outside the knowledge) of those on the ground. In order to facilitate this analysis, IE is geared toward exploring how lives are "put together across a multiplicity of different sites" (DeVault, 2008, p.6) by explicating "distinct social forms of coordination" (McCoy, 2008, p.703).

The first stage of analysis will aim to explicate the work that is happening on the ground in OSCEs; the micro-level. In the second stage, and characteristic of IE, the analytical focus will move to discover how this work is socially organised. Consideration may be given to producing maps of the relations within the institution, linking the micro to the macro if this is where the data leads. Smith suggests that the aim is "to develop a detailed, descriptive analysis of some portion of the institutional relations that have been identified as consequential, in order to show how these institutional work processes are organised and how they shape the ground of people's everyday experiences" (McCoy, 2008, p.121). Ultimately, the goal of the analysis is to facilitate those on the ground to have better control of the process and the possibility for change.

Institutional Ethnography is an empirical approach, where initial coding of the field notes and the observational data will take place, as work processes become apparent which will allow further exploration of these emerging ideas through the concurrent observations and interviews. In parallel, textual analysis will also be ongoing, informing and being informed by the other data collection methods. Both the computer software Nvivo and paper methods shall be used to organise and aid analysis of the data. 
The quality of the research in qualitative studies is measured in a very different way than would be typical in quantitative studies, for example in the COREQ checklist (Consolidated criteria for Reporting Qualitative research.) However, the quality of the research in IE is judged in a way that is quite distinct even amongst the broad umbrella of qualitative methods. Smith (2006) states that in order to serve those whose standpoint it is advocating for, the project must "produce accurate and faithful representations of how things actually work; it must be truthful" (p.42). In addition, reflexivity checks will be of utmost importance, increasing the rigour in keeping with this approach to qualitative research. This is achieved by critical cognisant of the position and assumptions of the researchers within the project and necessitates regular meetings of the research team to allow understanding and the analysis to evolve. Both reflective diaries and detailed notes of the research discussions will be kept to aid in this process.

\section{Personnel}

The Principal investigator of this study is $\operatorname{Dr} X X X$, who is an academic in the CME, XXX. Dr XXX is a $P h D$ student as well as a practising GP and sessional teacher/examiner. $\operatorname{Dr} X X X$ and $\operatorname{Dr} X X X$ are also academics working with CME, XXX. Dr XXX is an Adjunct Professor in the XXX whose published PhD used Institutional Ethnography. Data collection will primarily be conducted by XX, some data collection may also be undertaken by other members of the research team as in keeping with qualitative research. In this form of ethnographic research, variations in what different members of the team observe or learn in interview are welcomed as opportunities to broaden thinking and as a safe guard against becoming unknowingly involved in the institutional discourse at play. There is an emphasis on team reflexivity where each member is cognisant of their position in the research at each step of the process.

\section{Timeline}

The planned timescale for the research is June 2017 until September 2019, data collection and analysis will happen in parallel throughout this time.

\begin{tabular}{|c|c|c|c|c|c|c|c|c|c|c|c|c|c|c|c|c|c|c|c|c|c|c|c|c|c|c|c|c|}
\hline ACTIVITY & 20 & 17 & & & & & & 18 & & & & & & & & & & & & 19 & & & & & & & & \\
\hline & $\mathrm{J}$ & $\mathrm{J}$ & $A$ & $S$ & 0 & $\mathrm{~N}$ & $\mathrm{D}$ & $\mathrm{J}$ & $F$ & $M$ & $A$ & $M$ & J & J & $A$ & $\mathrm{~S}$ & 0 & $\mathrm{~N}$ & $\mathrm{D}$ & J & $\mathrm{F}$ & $M$ & $A$ & $M$ & J & J & A & $\mathrm{S}$ \\
\hline ETHICS & $x$ & & & & & & & & & & & & & & & & & & & & & & & & & & & \\
\hline PROCEDURES & $x$ & $x$ & $x$ & & & & & & & & & & & & & & & & & & & & & & & & & \\
\hline $\begin{array}{l}\text { DATA } \\
\text { COLLECTION }\end{array}$ & & & & $x$ & $\mathrm{x}$ & $x$ & $\mathrm{x}$ & $x$ & $x$ & $x$ & $x$ & $x$ & $x$ & & & & & & & & & & & & & & & \\
\hline ANALYSIS & & & & $x$ & $x$ & $x$ & $x$ & $x$ & $x$ & $x$ & $x$ & $x$ & $x$ & $x$ & $x$ & $x$ & & & & & & & & & & & & \\
\hline WRITE UP & & & & & & & & & & & & & & & & $x$ & $x$ & $x$ & $x$ & $x$ & $x$ & $x$ & $x$ & $x$ & $x$ & $x$ & $x$ & $x$ \\
\hline
\end{tabular}

\section{Ethics}


This study received ethical approval from the School of Medicine, Dentistry and Biomedical Sciences, Ethics Committee at Queen's University Belfast (Ref: 17.29v2.) Participants will be emailed a "Participant Information Leaflet" in advance of any data collection, allowing them the opportunity to consider and withhold consent if requested; written consent will be obtained for those who will be directly observed and interviewed. Any participant who does not give consent will not be observed either as part of the direct or general observation. Participants are free to withdraw from the project at any stage and all potential participants will be advised that their wish not to participate will not adversely affect their employment, career progression or relationship with the research team.

The presence of the researcher at any of the meetings or on the day(s) of the OSCE will be as unimposing as possible to minimise any influence on the process. No "live" OSCE stations will be directly observed, eliminating any risk of impacting performance at the station either positively or negatively.

All data collected will be treated securely, anonymously and confidentially as per XXX research practice policies. Strict confidentiality will also be maintained on any examination content or outcome. Safeguards will be put in place to protect the confidentiality of all participants with password protected anonymous data held on secured databases for the duration of the project. Any images obtained will not contain anything that could allow identification of any participant or breach any examination security.

\section{Acknowledgments}

The study fees and maintenance for this project come from a Research Studentship from the Northern Ireland Department for the Economy (DfE). The team would like to thank all those who participated in this study for their time and expertise.

\section{References}

Campbell, M., \& Gregor, F. (2002). Mapping social relations: A primer in doing institutional ethnography. Toronto: Garamond Press.

Corman, M. K. (2017). Paramedics on and off the streets: Emergency medical services in the age of technological governance. Toronto: The University of Toronto Press 
DeVault, 2008. (Ed.). 2008. People at work: Life, power, and social inclusion in the new economy. U.S.A.: New York University Press.

DeVault, M., \& McCoy, L. (2006). Institutional ethnography: 'Using interviews to investigate ruling relations. In. D. Smith (Ed.), Institutional ethnography as practice (pp. 15-44). U.S.A.: Rowman \& Littlefield Publishers, Inc.

Diamond, T. (1992). Making gray gold: Narratives of nursing home care. Chicago, Illinois: The University of Chicago Press.

Emmerson, R., Fretz, R., \& Shaw, L. (2011). Writing ethnographic fieldnotes ( ${ }^{\text {nd }}$ ed.) Chicago \& London: The University of Chicago Press.

Gormley, G., Hodges, B., McNaughton, N., \& Johnston, J. (2016). The show must go on? Patients, props and pedagogy in the theatre of the OSCE. Medical Education, 50, 1237-1240.

Khan, K., Gaunt, K., Ramachandran, S., \& Pushkar, P. (2013). AMEE Guide no 81. The Objective Structured Clinical Examination. Part 1: an historical and theoretical perspective. Medical Teacher, 35, 1437-46.

McCoy, L. (2006). Keeping the institutional in view: Working with interview accounts of everyday experience. In D Smith (Ed.) Institutional ethnography as Practice. (pp 109-125). U.S.A.: Rowman \& Littlefield Publishers, Inc.

McCoy, L. (2008). Institutional ethnography and constructionism. In J. Hostein \& J. Gubrium (Eds.) Handbook of constructionist research (pp. 701-714). New York: Guilford.

Rankin, J., \& Campbell, M. (2006). Managing to nurse: Inside Canada's health care reform. Canada: University of Toronto Press.

Shay, S. (2008). Researching assessment as social practice: Implications for research methodology. International Journal of Educational Research, 47, (3) 159-164.

Shepard, L. (2000). The Role of Assessment in a Learning Culture. Educational Researcher, 29 (7) 414.

Smith, D. (1987). The Everyday World As Problematic: A Feminist Sociology. Boston, USA: Northeastern University Press

Smith, D. (2005). Institutional ethnography: A sociology for people. Toronto: AltaMira Press.

Smith, D. (Ed.) (2006). Institutional ethnography as practice, Maryland, USA: Rowman \& Littlefield Publishers, Inc.

Zaidi, S., \& Nasir, M. (2015). Assessment and Evaluation. In S. Zaidi, \&M. Nasir (Eds.) Teaching and Learning Methods in Medicine (pp. 109-135). Springer, Cham. 
